\title{
An Enhanced Bidimensional Empirical Mode Decomposition Algorithm Based on Wavelet Transform for SAR Internal Solitary Images
}

\author{
Guang-Mei WEI \\ LMIB and School of Mathematics and System Sciences \\ Beihang University, \\ Beijing, 100191, China \\ E-mail: gmwei@buaa.edu.cn
}

\author{
Bing LI, Xing-Xing NIE \\ School of Computer Science and Engineering \\ Beihang University, \\ Beijing, 100191, China \\ E-mail: libing@nlsde.buaa.edu.cn \\ niexingxing@nlsde.buaa.edu.cn
}

\begin{abstract}
Synthetic aperture radar (SAR) produces ocean SAR solitary images which include the information of internal solitary waves (ISWs), surface wave, terraininfo and noise. Based on wavelet transform (WT) and bidimensional empirical mode decomposition (BEMD), we give a new algorithm, named wBEMD, to obtain information of ISWs from the SAR images, and apply it on two SAR images to extract ISWs features. The ISWs information layer obtained from wBEMD shows more intact ISW features, from which we retrieve the characteristic width and phase velocity of ISWs and analyse other detailed information. The closeness between the retrieved parameters and observed data verifies wBEMD's practicability.
\end{abstract}

Keywords-SAR image; Internal solitary wave; Wavelet transform; Bidimensional empirical mode decomposition

\section{INTRODUCTION}

Internal solitary waves (ISWs), different from normal linear internal tide wave, are non-linear large amplitudes internal waves in marine pycnocline, which may be generated by the full variety of mechanisms forcing currents over the continental shelf and slope $[1,2]$. ISWs are extremely important in marine underwater engineering, underwater navigation and marine petroleum exploitation et al. [3]. The northern South China Sea (SCS) is one of the waters where ISWs appear frequently [4]. The most widely used mathematical models in this water are Korteweg-de Vries $(\mathrm{KdV})$ equation and its reformed or extended forms, such as extended $\mathrm{KdV}(\mathrm{eKdV})$ equation, modified $\mathrm{KdV}$ $(\mathrm{mKdV})$ equation, and Regularized Long Wave (RLW) equation [5-13].

Plenty of researches on ISWs in northern SCS have been carried out for the SAR images generated by satellites ERS1/2 SAR, ENVISAT, RADARSAT [14, 15]. The observed data we use in this paper come from the the Asian Seas International Acoustics Experiment (ASIAEX) [15].

The radar signatures of internal waves have double sign, which means that the corresponding radar image consists of bright and dark streaks associated respectively with enhanced and reduced radar reflectivity compared with the local mean [16]. There are several methods to extract ISWs features, such as Fourier transform (FT), wavelet transform (WT) [17-19] and empirical mode decomposition (EMD) $[20,21]$. FT has advantage in obtaining the main wave information, whereas information of wave group is omitted, and WT can get wave shape of internal wave. However, the selection of wavelet basis has great impact on processing result [22]. EMD algorithm was firstly proposed by Huang in 1998 and modified in 1999 [23, 24], which has a well performance in multiscale decomposition and is widely used in extracting internal wave information from ocean SAR images [20]. Bimensional empirical mode decomposition (BEMD) algorithm is a two dimensional algorithm improved from original EMD [21]. In order to extract more intact ISWs features, we propose an enhanced BEMD algorithm based on WT.

This paper is organized as follows. Section 2 introduces the particular analytical model retrieving the characteristic width and the phase velocity of ISWs. Section 3 provides SAR radar imaging of internal waves, the WT method, BEMD algorithm and our improved algorithm. We present the result of experiments and the comparison with observed data in Section 4. The conclusion are given in Section 5.

\section{MODEL FOR ISW AND DETERMINATION OF THE CHARACTERISTIC PARAMETERS}

Synthetic aperture radar (SAR) has brought about the realization of the widespread existence of ISWs and provided the major impetus for study. However, compared with in-situ observations, there are less ISW parameters that can be obtained directly from the SAR image [25]. Previous studies indicated that some ocean ISW parameters could be determined directly with a SAR image or a time series of SAR images, which includes the wavelength or separation distance between two consecutive waves, length of crest line [26]. For SAR images with ISW groups, the group velocity can be calculated as below,

$$
C_{g}=\frac{\Lambda}{T}
$$

Where $C_{g}$ is the group velocity, $\Lambda$ is the distant between ISW groups, and $T$ is semidiurnal tide cycle [27]. In general, the phase velocity of an ISW is considered equally to the group velocity of the ISW groups.

Some important parameters, such as characteristic width which determines the shape and the amplitude, can not be obtained directly from the SAR image [26]. The main method of retrieving ISWs parameters from the SAR image is under the framework of weak nonlinear, weak dispersion approximation (ISWs theory) [28]. Two-layer KdV model is 
a typical example, and is used to describe the ISWs in SCS, with which we get relative change of image gray value caused by ISWs as below,[29]

$$
\frac{\Delta I}{I_{0}}=\frac{\Delta \sigma}{\sigma_{0}}=B \operatorname{sech}^{2}\left(\frac{x^{\prime}}{\lambda / 2}\right) \tanh \left(\frac{x^{\prime}}{\lambda / 2}\right),
$$

where $B= \pm \frac{4+\gamma}{\mu} \frac{2 C_{0} \eta_{0}}{h_{1}(\lambda / 2)} \cos ^{2} \phi, I_{0}$ is background sea gray value, $\Delta I$ is the difference of ocean surface gray value, $\sigma_{0}$ is the background normalized radar cross section (NRCS), $\Delta \sigma$ is the NRCS difference between the ISWs field and background field, $x^{\prime}=x-C_{p} t$, and $\lambda$ is internal wave characteristic width.

We obtain $x^{\prime}= \pm 0.33 \lambda$ from Eq. (2), the characteristic width of an ISW $\lambda$ can be determined by

$$
\lambda=\frac{D}{0.66} .
$$

\section{METHODS OF PROCESSING SAR IMAGE}

Synthetic aperture radar (SAR) maps an area by transmitting pulses of microwave energy. According to SAR imaging theory, a SAR image contains not only information of the ocean surface currents affected by ISWs, sea surface winds and pressure fields, but also some speckle noises [30]. In order to extract precise oceanic ISWs features, we have to process SAR images into various resolutions by using different scale of processing [31-33], such as WT and BEMD.

\section{A. Wavelet transfrom analysis}

Wavelet transfrom is inherently localized in both time and frequency, and has been widely used in the denosing field because of its characteristics, such as low entropy, multi-resolution, decorrelation and flexibility wavelet choosen [34, 35]. Wavelet soft thresholding denoising algorithm was firstly proposed in 1995 by Donoh, who proved the effectiveness of the algorithm and gave the calculation method of threshold [36]. We improve Donoh's algorithm from one threshold to selecting thresholds in different scales and directions.

\section{B. Bidimensional Empirical Mode Decomposition Algorithm}

The EMD was introduced in signal processing by Huang, BEMD algorithm is the developed EMD algorithm, and is mainly used in multi-scale decomposition of the image.

BEMD algorithm breaks down an image to a series of 2D Intrinsic Mode Functions (IMFs), the process of BEMD is almost the same as EMD, and the main difference is the sifting process, which is bidimensional sifting process. Bidimensional sifting process is usually proceeded for several times, which repeated by BEMD until there is no more 2D IMF.

\section{The Enhanced BEMD algorithm}

SAR images are inevitably affected by speckle noise, due to the coherent nature of scattering phenomena and corrupting the images' feature [37]. The first step in BEMD algorithm is to calculate local extremes of the image, which are related to the scatter speckle noise. Thus, it appears sensible to reduce speckle in SAR images without losing the edges and textural information.

The speckle in SAR images is generally modeled as multiplicative random noise [38]. It is necessary to apply a logarithmic transform to convert the multiplicative model into an additive one [30]. Next an w BEMD algorithm will be proposed, which combines WT and BEMD algorithms. The detailed algorithm is as follows.

a) Make a logarithmic transformation of the original image $X$,

b) Use wavelet soft threshold mentioned in Section 3.1 on log-transformed image,

c) Invert the logarithmic transformation and get the de-speckle image $Y$,

d) Decompose the de-speckle imageY tonIMFs using the BEMD algorithm mentioned in Section 3.2,

e) Calculate the normalized deflection of each IMF based on the formule,

$$
\sigma_{i}=\frac{v_{i}^{2}}{\sum_{i=1}^{i=n} v_{i}^{2}},
$$

where $v_{i}^{2}$ is the standard deviation of the $I M F_{i}$.

The bigger the normalized deflection is, the more energy the signal contains [39]. The IMF which contains the largest normalized deflection is the contained ISW signal layer.

\section{EXPERIMENT ON TWO SAR IMAGES}

\section{A. Experiment on an ERS-2 SAR image}

One SAR image, taken by ERS-2 over northern SCS at 02:44 on 27th May 1998, will be selected in this subsection, from which we cut a $298 * 243$ subimage, see Figure 1 . The subimage's center spot is located at $116^{\circ} 38^{\prime} 01^{\prime \prime} \mathrm{E}, 20^{\circ} 35^{\prime} 41^{\prime \prime}$ $\mathrm{N}$, northern SCS, with the actual distance between the two adjacent pixels is 12.5 meters.

Figure 1 (b) shows obvious ISWs information with various other scales information, such as speckle noise, sea surface and terrain wave and so on. According to Section 2.4, characteristic width of ISW can be retrieved if we have the distance, we need to get relatively intact ISW's wave shape by extracting sole solitary texture information. The wave group velocity can be obtained from the distance between two consecutive wave groups aroused by the same excitation source.

The results of SAR image decomposing methods are described as follows. Figure 1 shows the original SAR image taken in 1998 and its subimage, in which (a) shows the original SAR image's geographic location, and the arrow $\mathrm{AB}$ shows the propagation path of the wave groups, we cut a subimage in the blue transparent box whose details are shown in Figure 1 (b). 


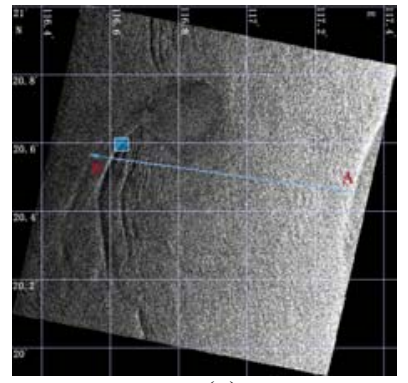

(a)

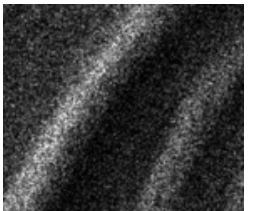

(b)

Figure 2. (a) ERS-2 SAR image taken at 02:44, on 27th May 1998, SCS; (b) a subimage of (a).

The solitary texture information after wavelet threshold denoising have been shown in Figure 2 (a), where most of noise on the surface have been wiped out. However, some smaller scales information, other than ISWs, still affects the extraction of sole solitary texture. As shown in Figure 2 (b), different scale information layers have been obtained from BEMD algorithm. The difficulty of parameter's retrieve caused by overlying of other scales information have been overcome by multiscale information separation. But there are some distortions emerging in the result image, we need to improve BEMD algorithm.

The wBEMD algorithm wipes out the speckle noise by WT before extracting the sole solitary texture information by using BEMD algorithm, see Figure 2 (c). Some distortions and other scales information have been eliminated, and intact ISWs shapes have been obtained.

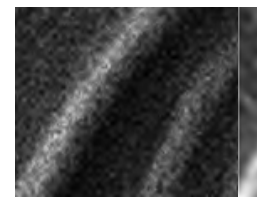

(a)

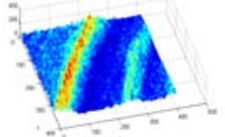

(d)

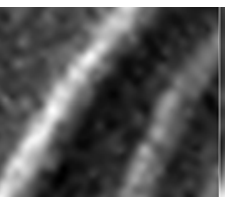

(b)

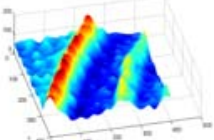

(e)

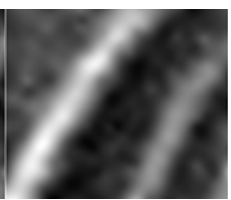

(c)

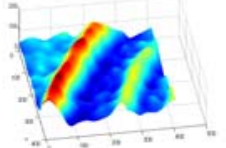

(f)
Figure 3. (a) the result after wavelet threshold denoising of Figure 1 (b); (b) the result after BEMD decomposition; (b) the result after wBEMD decomposition; (d)(e)(f) 3D profile of (e).

Ten curves and the arrow $\mathrm{AB}$ have been selected in SAR image to calculate $D$ and $\Lambda$. With Eq. (1) and Eq. (3), we can get the characteristic width $\lambda$ and phase velocity $C_{p}$ of ISWs.

As shown in Table I, two 4th layers represent the ISWs information in the two methods and relatively more proportion of ISWs information obtained in the wBEMD algorithm, which have been shown in Figure 2.
TABLE I. NORMALIZED VARIANCE IN FIGURE 2

\begin{tabular}{|l|c|c|c|c|}
\hline Methods & 1st layer & 1st layer & 3rd layer & 4th layer \\
\hline BEMD & 0.3182 & 0.3182 & 0.0042 & 0.6538 \\
\hline wBEMD & 0.1124 & 0.0158 & 0.0052 & 0.8665 \\
\hline
\end{tabular}

\section{B. Experiment on an ENVISAT SAR image}

The SAR image we select in this subsection was taken by ENVISAT SAR over northern SCS at 02:18, on 22th July 2011. Figure 3 shows the original image for our experiment, whose center spot is located at $117^{\circ} 09^{\prime} 32^{\prime \prime} \mathrm{E}, 21^{\circ} 19^{\prime} 27^{\prime \prime} \mathrm{N}$, Dongsha Islands, northern SCS. We cut out a 298*243 subimage, which contains abundant ISWs information with obvious ISWs features in leading waves, see Figure 3 (b). The actual distance between the two adjacent pixels is 75

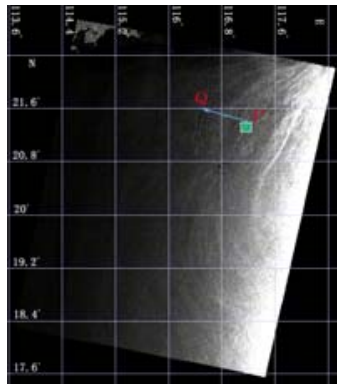

(a)

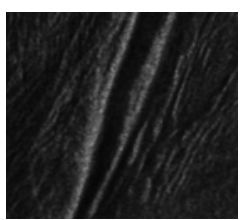

(b)
Figure 1. (a) ENVISAT SAR image taken at 02:18, on 22th July 2011, SCS; (b) a subimage of (a).

meters. The image shows various scales information, such as speckle noise, sea surface wave, terrain, ISWs and so on.

The results of SAR image decomposing methods are described as follows. Figure 3 shows the original SAR image and its subimage, in which (a) shows the original SAR image's geographic location, and the arrow PQ shows the propagation path of the wave groups. Details of subimage in the green transparent box are shown in Figure 3 (b).

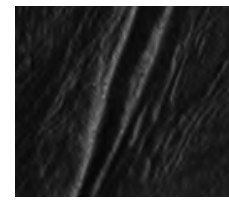

(a)

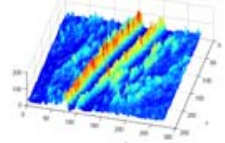

(d)

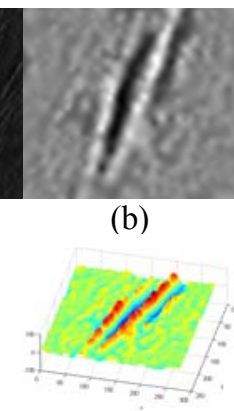

(e)

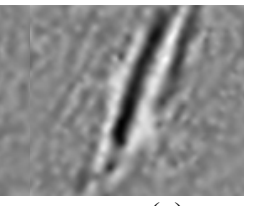

(c)

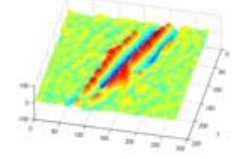

(f)
Figure 4. (a) the result after wavelet threshold denoising of Figure 3 (b); (b) the result after BEMD decomposition; (b) the result after wBEMD decomposition; (d)(e)(f) 3D profile of (e). 
In Figure 4, (a), (b), (c) show the solitary texture information after wavelet threshold denoising, BEMD algorithm decomposition and the wBEMD algorithm decomposition, (d), (e), (f) represent their 3D profiles respectively.

Ten curves and the arrow PQ have been selected in the SAR image to calculate the characteristic width $\lambda$ and phase velocity $C_{p}$ with Eq. (1) and Eq. (3).

As shown in Table II, the third and the second layers represent the ISWs information in two methods respectively, and relatively more proportion of ISWs information is obtained by wBEMD algorithm, see Figure 4.

TABLE II. NORMALIZED VARIANCE IN FIGURE 4

\begin{tabular}{|l|c|c|c|c|}
\hline Methods & 1st layer & 1st layer & 3rd layer & 4th layer \\
\hline BEMD & 0.0696 & 0.1529 & 0.4715 & 0.3060 \\
\hline WBEMD & 0.2595 & 0.6158 & 0.0348 & 0.0899 \\
\hline
\end{tabular}

\section{Analysis of experimental results}

As shown in Figure 2 and Figure 4, more ISWs information have been retained in the decomposition results of wBEMD algorithm with less others scales information and distortion, from which we can retrieve accurate ISW parameters.

Figure 5 shows that gray profile curves have been obtained deriving from 10 selected curves in the two SAR subimages, by which we can use the average value of $D$ to calculate characteristic width of ISWs. Due to the roughness and oscillation of gray profile after WT, we only calculate the values of $D$ from BEMD algorithm and wBEMD algorithm, which have been shown in Table III.

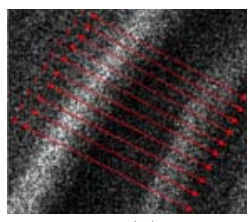

(a)

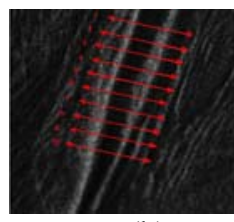

(b)
Figure 5. (a) 10 data lines on the ERS-2 SAR subimage; (b) 10 data lines on the ENVISAT SAR subimage.

The average values of $D$ that we have got in Table III are agreed with each other, which implies wBEMD algorithm is more feasible in SAR image processing. The observed data about ERS-2 SAR image that we choose in this paper were obtained in 10th May, 2001. Since the ISWs generation process varies on seasonal [40], and the imaging time of our first SAR image is same with the obtained time of observed data in months, which enables the retrieved parameters can be compared to the observed data to verify their practicability. Table VI and Table V present the comparison of experimental data.
TABLE III $\quad$ THE DISTANCE BETWEEN THE BRIGHTEST AND THE DARKEST SPOTS

\begin{tabular}{|l|c|c|c|c|}
\hline \multirow{2}{*}{ D } & \multicolumn{2}{|c|}{ Figure 5 (a) } & \multicolumn{2}{c|}{ Figure 5 (b) } \\
\cline { 2 - 5 } & BEMD & wBEMD & BEMD & wBEMD \\
\hline 1 & 101.8 & 74.7 & 27.6 & 17.3 \\
\hline 2 & 85.6 & 73.5 & 18.5 & 19.5 \\
\hline 3 & 66.4 & 72 & 21.5 & 21.6 \\
\hline 4 & 99.7 & 81 & 27.7 & 21.6 \\
\hline 5 & 76.8 & 82 & 24.6 & 23.6 \\
\hline 6 & 82.7 & 69.5 & 24.6 & 19.5 \\
\hline 7 & 98 & 97.6 & 24.6 & 19.5 \\
\hline 8 & 74.8 & 71 & 22.5 & 20.5 \\
\hline 9 & 71.4 & 74.5 & 19.45 & 31.4 \\
\hline 10 & 74.7 & 86 & 21.4 & 17.5 \\
\hline average & 83.19 & 78.18 & 23.245 & 21.39 \\
\hline $\begin{array}{l}\text { actual } \\
\text { distance (m) }\end{array}$ & 1039.88 & 977.25 & 1743.38 & 1604.25 \\
\hline
\end{tabular}

TABLE IV. THE PHASE VELOCITY OF ISWS

\begin{tabular}{|c|c|c|c|}
\hline $\boldsymbol{C}_{\boldsymbol{p}}$ & $\boldsymbol{\Lambda}(\boldsymbol{m})$ & $\boldsymbol{C}_{\boldsymbol{p}}(\boldsymbol{m} / \boldsymbol{s})$ & $\begin{array}{c}\text { Observed data } \\
(\boldsymbol{m} / \mathbf{s})\end{array}$ \\
\hline Figure 1 (b) (AB) & 81760 & 1.817 & 1.24 \\
\hline Figure 3 (b) (PQ) & 75178 & 1.67 & no data \\
\hline
\end{tabular}

TABLE V. THE CHARACTERISTIC WIDTH OF ISWS

\begin{tabular}{|l|c|c|c|}
\hline \multicolumn{1}{|c|}{$\boldsymbol{\lambda}$} & BEMD & wBEMD & $\begin{array}{c}\text { Observed data } \\
(\boldsymbol{m})\end{array}$ \\
\hline Figure 1(b) & 1575.57 & 1480.68 & 1500 \\
\hline Figure 3(b) & 2641.48 & 2430.68 & no data \\
\hline
\end{tabular}

\section{CONCLUSION}

Spackle noise in SAR image makes a challenge to extract the sole solitary texture information accurately from SAR image. In this paper, we have proposed an enhanced BEMD algorithm based on WT, which combines the advantages of WT and BEMD methods and remedy the disadvantages in each method. Experimental results have shown that wBEMD algorithm can get better sole solitary texture information than WT method and reduce the effect of spackle noise on decomposition result in each layer. The retrieved parameters obtained from the improved algorithm in ERS-2 image are very closed to the observed data, which indicates our algorithm is more practical. However, the decomposition results depend on the selection of threshold values and wavelet basis, which is our further research interest.

\section{ACKNOWLEDGMENT}

This work has been supported by the National Natural Science Foundation of China under Grant No. 61072145. 


\section{REFERENCES}

[1] M. H. John. Circulation, exchange and water masses at the ocean margin: the role of physical processes at the shelf edge. Progress in Oceanography, 1995, 35, pp.353-431.

[2] R. Pingree, G. Mardell. Solitary internal waves in the celtic sea. Progress in O-ceanography, 1985, 14, pp.431-441.

[3] H. Sandstrom, J. A. Elliott. Internal tide and solitons on the scotian shelf: A nutrient pump at work. Journal of Geophysical Research, 1984, 89, pp.6415-6426.

[4] A. K. Liu, Y. S. Chang, M. K. Hsu, N. K. Liang. Evolution of nonlinear internal waves in the east and south china seas. Journal of Geophysical Research, 1998, 103, 7995-8008.

[5] P. T. Shaw, D. S. Ko, S. Y. Chao. Internal solitary waves induced by flow over a ridge: With applications to the northern south china sea. Journal of Geophysical Research, 2009, 114

[6] T. Du, Y. H. Tseng, X. Yan. Impacts of tidal currents and kuroshio intrusion on the generation of nonlinear internal waves in luzon strait. Journal of Geophysical Research, 2008, 113.

[7] T. Du. Studies of internal waves with large amplitude in shallow strong pycnoline. Earth Science Frontiers, 2000, 7, pp.179-188.

[8] A. K. Liu, S. R. Ramp, Y. Zhao, T. Y. Tang. A case study of internal solitary wave propagation during asiaex 2001. IEEE Journal of Oceanic Engineering, 2004, 29, pp.1144-1156.

[9] S. Y. Chao, D. S. Ko, R. C. Lien, P. T. Shaw. Assessing the west ridge of luzon strait as an internal wave mediator. Journal of Oceanography, 2007, 63, pp.897-911.

[10] Z. S. Fan, Y. L. Zhang, M. Song. A study of sar remote sensing of internal solitary waves in the north of the south china sea: I. simulation of internal tide transformation. Acta Oceanologica Sinica, 2008, 27, pp.36-48.

[11] S. Q. Cai, J. S. Xie. A propagation model for the internal solitary waves in the northern south china sea. Journal of Geophysical Research, 2010, 115.

[12] P. J. Lynett, P. L. F. Liu. A two-dimensional, depth-integrated model for internal wave prop-agation over variable bathymetry. Wave Motion, 2002, 36, pp.221-240.

[13] S. Y. Chao, P. T. Shaw, M. K. Hsu. Reflection and diffraction of internal solitary waves by a circular island. Journal of Oceanography, 2006, 62, pp.811-823.

[14] M. K. Hsu, A. K. Liu. Nonlinear internal waves in the south china sea. Canadian Journal of Remote Sensing, 2004, 26, pp.72-81.

[15] F. L. James, H. D. Peter. Overview of asiaex field experiments in the south and east china seas. Jounal of the Acoustical Society of America, 2002, 112, pp.2360-2361.

[16] W. Alpers. Theory of radar imaging of internal waves. Nature, 1985, 314 , pp. $245-247$.

[17] J. A. Rodenas, R. Garello. Wavelet analysis in sar ocean image profiles for internal wave detection and wavelength estimation. IEEE Transactions on Geoscience and Remote Sensing, 1997, 35, pp.933945

[18] J. A. Rodenas, R. Garello. Internal wave detection and wavelength estimation from ERS-1 SAR images via wavelet analysis. Seventh International Conference on Electronic Engineering in Oceanography, Technology Transfer from Research to Industry. 1997, pp.238-245.

[19] J. A. Rodenas, R. Garello. Internal wave detection and location in SAR images using wavelet transform. IEEE Transactions on Geoscience and Remote Sensing. 1998, 36, pp.1494 -1507.

[20] X. L. Gan, W. G. Huang, J. S. Yang. A new method to extract internal wave parameters from sar imagery with hilbert-huang transfrom. Journal of Remote Sensing, 2007, 11, pp.39-47.
[21] J. Kang, J. Zhang, P. J. Song, J. M. Meng. The application of twodimensional emd to extracting internal waves in sar images. In 2008 International Conference on Computer Science and Software Engineering. 2008, 1: $953-956$.

[22] Y. Arvelyna, M. Oshima. New application of wavelet transform for internal wave detection sar and optical image: A case study in japan waters. In OCEANS 2007 -Europe(pp. 1-6).

[23] N. E. Huang, Z. Shen, S. R. Long. The empirical mode decomposition and the hilbert spectrum for nonlinear and non-stationary time series analysis. Proceedings of the Royal Society A: Methematical, Physical, \&amp; Engineering Sciences, 1998, 454, pp.903-995.

[24] N. E. Huang, Z. Shen, S. R. Long. A new view of nonlinear water waves: the hilbert spectrum. Annual Review of Fluid Mechanics, 1999, 31, pp.417-457.

[25] C. Jackson, J. Apel. Synthetic aperture radar marine user's manual. (pp. 189-201). Washington D C: National Environmental Satellite, 2004.

[26] Q. Zheng, Y. Yuan, V. Klemas, X. H. Yan. Theoretical expression for an ocean internal soliton synthetic aperture radar image and determination of the soliton characteristic half width. Journal of Geophysical Research, 2001, 106, pp.31415-31423.

[27] J. Zhong, Y. Y. Ou, F. Li, M. H. Zhu. Synthetic aperture radar images of ocean internal wave detection. (1st ed.). Beijing: Ocean Press. 2010.

[28] I. Liu. Satellite Remote Sensing of South China Sea. world scientific. 2008.

[29] J. Zhang. Synthetic aperture radar on marine information processing and application. Beijing: Science Press. 2004.

[30] L. Gagnon, A. Jouan. Speckle filtering of sar images - a comparative study between complex-wavelet-based and standard filters. Proceedings of SPIE, 1997, 3169, pp.80-91.

[31] D. R. Thompson, R. F. Gasparovie. Intensity modulation in sar images of internal waves. Nature, 1986, 320, pp.345 - 348.

[32] P. Brandt, R. Romeiser, A. Rubino. On the determination of characteristics of the interior ocean dynamics from radar signatures of internal solitary waves. Journal of Geophysical Research, 1999, 104, pp.30039-30045.

[33] da Silva J C B, S. A. Ermakov, I. S. Robinson. Role of surface films in ers sar signatures of internal waves on the shelf: 3 . mode transitions. Journal of Geophysical Research, 2000, 105, pp.24089-24104.

[34] B. Vidakovic, C. Lozoya. On time-dependent wavelet denoising. IEEE Transactions on Signal Processing, 1998, 46, pp.2549-2554.

[35] S. Ruikar, D. Doye. Image denoising using wavelet transform. In 2010 2nd Interna-tional Conference on Mechanical and Electrical Technology (ICMET)(pp. 509-515).

[36] D. Donoho. De-noising by soft-thresholding. IEEE Transactions on Information Theory, 1995, 41, 252, pp.613-627.

[37] D. Gnanadurai, V. Sadasivam, J. Nishandh, L. Muthukumaran, C. Annamalaie. Undecimated double density wavelet transform based speckle reduction in sar images. Computers \&amp; Electrical Engineering, 2009, 35, pp.209-217.

[38] H. Xie, L. Pierce, F. Ulaby. Statistical properties of logarithmically transformed speckle. IEEE Transactions on Geoscience and Remote Sensing, 2002, 40, pp.721 -727.

[39] J. Rodenas, R. Garello. Internal wave packet characterization from sar images us-ing wavelet transform modulus maxima (wtmm). In Geoscience and Remote Sensing Symposium Proceedings, 1998. IGARSS '98. 1998 IEEE International(pp. 930 -932 vol.2). volume 2.

[40] J. R. Apel. A new analytical model for internal solitons in the ocean. Journal of Physical Oceanography, 2003, 33, pp.2247-2269. 